\title{
Primary cilia and their role in cancer (Review)
}

\author{
MICHAEL HIGGINS, ISMAEL OBAIDI and TARA MCMORROW
}

School of Biomolecular and Biomedical Science, Conway Institute, University College Dublin, Belfield, Dublin 4, Ireland

Received November 6, 2017; Accepted January 24, 2018

DOI: $10.3892 / \mathrm{ol} .2019 .9942$

\begin{abstract}
Primary cilia are microtubule-based organelles that are expressed on almost all mammalian cells. It has become apparent that these structures are important signaling hubs that serve crucial roles in Wnt, hedgehog, extracellular signal-regulated kinase $($ ERK)/mitogen-activated protein kinase (MAPK) and Notch signaling pathways. A number of diseases have been found to involve dysfunctional primary cilia; collectively these diseases are called ciliopathies. In recent years, there has been more focus on the association between primary cilia and cancer, including renal, pancreatic and breast cancer. Numerous studies have demonstrated that various types of cancer cells fail to express cilia. Notably, it has also been indicated that a number of renal carcinogens induce a significant loss of cilia in renal epithelial cells. The present review focuses on the existing literature regarding primary cilia and their involvement with cancer signaling pathways, providing a brief overview of the structural features and functions of primary cilia, then discussing the evidence associating primary cilia with cancer, and presenting the available information on the ERK/MAPK, hedgehog and Wnt signaling pathways, and their involvement in primary cilia in association with cancer.
\end{abstract}

\section{Contents}

1. Introduction

2. Ciliopathies

3. Primary cilia in the cell cycle

4. The loss of primary cilia expression in cancer

5. Primary cilia and ERK/MAPK signaling in cancer

6. Primary cilia and hedgehog signaling in cancer

7. Primary cilia and Wnt signaling in cancer

Correspondence to: Dr Tara McMorrow, School of Biomolecular and Biomedical Science, Conway Institute, University College Dublin, Room F072, Belfield, Dublin 4, Ireland

E-mail: tara.mcmorrow@ucd.ie

Key words: primary cilia, cancer, ciliopathies, cancer, cancer signaling pathways

\section{Introduction}

The primary cilium is an immotile microtubule-based structure that protrudes from the surface of almost all mammalian cells (1). The primary cilium is comprised of a $9+0$ microtubule arrangement ( 9 outer microtubules with no inner microtubules), differing from the $9+2$ arrangement of motile cilia, thus lacking a central pair of microtubules (Fig. 1) (1). The axoneme is anchored to the cell by the basal body, which is nucleated by the mother centriole. The mother centriole is crucial for the formation of the centrosome, which acts as the major microtubule-organizing center (MTOC) in mammalian cells (2). In general the presence of cilia are dynamically regulated during the progression of the cell cycle (Fig. 2), they are present during $G_{0}$ and $G_{1}$, and usually in $S / G_{2}$, but are generally resorbed prior to entry into mitosis (3). Therefore, there is an inverse association between the presence of the primary cilium and cell proliferation, if the cell is dividing the cilium is resorbed into the cell and the centrosome can act as the mitotic pole (4). Resorption is the process by which the cilium retracts into the cell, this usually precedes mitosis and the re-entry of cells into the cell cycle (5). Resorption is thought to involve the regulation of intraflagellar transport (IFT), the process that controls the growth and maintenance of the cilium and the de-acetylation of axonemal microtubules (5). The cilium is formed and maintained by the transport of proteins up the axoneme, via the IFT process (6). Trafficking to the cilium is a highly coordinated process. IFT trafficking from the base of the cilium right to its tip is heavily dependent on kinesin superfamily proteins (KIFs) (7). KIF3 is one of the most highly expressed KIFs in mammalian cells, which is crucial for ciliogenesis and cilia maintenance (7). It associates with two IFT complexes, IFT-A and IFT-B. IFT-B has been determined to be essential for anterograde trafficking; therefore, it associates with KIF3 to transport vesicles from the cell body to the tip of the cilium (7). Whilst IFT-A was determined to be crucial for retrograde trafficking, which involves channeling vesicles from the tip of the cilium towards the cell body (7).

It has now become apparent that the primary cilium has a diverse range of functions. The cilium has been demonstrated to be a crucial signal transduction hub, involving signaling pathways with relevance to development and diseases, including polycystic kidney disease and cancer (8). The primary cilium has a number of signaling receptors localized to it, including receptors for sonic hedgehog (Shh), Notch canonical and non-canonical Wnt signaling (9-11). Several components of the 
Wnt signaling pathway have been determined to localize to the cilium, including Frizzled3, Dishevelled2 (Dsh), $\beta$-catenin and glycogen synthase kinase- $3 \beta$ (11). Notably, mouse embryos with knockouts of a number of ciliary proteins, including KIF3a (controls ciliary formation), exhibit abnormal $\beta$-catenin and dysfunctional canonical Wnt responses (12). Hedgehog signaling is crucial for embryo development and cell proliferation. Another study has also shown that IFT88 and KIF3a, both cilia-associated genes are required for hedgehog signaling (13). Other hedgehog components, including Patched (Ptch)1, smoothened (Smo), glioma-associated oncogene family zinc finger (Gli) and suppressor of fused have all been confirmed to be enriched in cilia (13-15). Notch receptors have been indicated to co-localize to the primary cilium (16). Notch signaling serves a role in cell fate and cell-cell communication. Differentiation defects in ciliary mutants were rescued by Notch activation, indicating the important role Notch signaling serves in cilia (16).

Planar cell polarity (PCP) is the polarization of a field of cells. Epithelial cells possess PCP where structures, including tight junctions, desmosomes and adherens junctions, allow the formation of the epithelial layer (17). Studies have demonstrated a connection between the cilium and PCP $(18,19)$. Patel et al investigated acute kidney-induced cyst formation in KIF3a-mutant mice, which induced cilia loss while also causing aberrant PCP and abnormalities in cell division (18).

\section{Ciliopathies}

The important role of the primary cilium is becoming more apparent with the emergence of a growing list of diseases collectively called ciliopathies. These diseases all have abnormal ciliary functions. Autosomal dominant polycystic kidney disease (ADPKD) was the first ciliopathy to be identified. ADPKD is an inherited condition, which causes the over-proliferation of epithelial cells, leading to cyst formation (19). These cysts cause damage throughout the kidney and eventually progress to end-stage renal disease (20). ADPKD is caused by a mutation in polycystin-1 (PC-1) or polycystin-2 (PC-2), these are both required for the normal development of renal tubules and are localized in the cilium (21). Nephronophthisis (NPHP) is a condition characterized by corticomedullary cysts, tubular basement membrane disruption and tubulointerstitial nephropathy, and is the leading cause of chronic kidney disease in people aged $<30$ years (22). Although a single mutation is sufficient to cause NPHP, it has been determined that $>1$ gene can cause NPHP (22). Collectively these genes are called nephrocystins, and have been determined to localize in the primary cilium, the basal body and the centrosome. Bardet-Biedl Syndrome (BBS) is another well-known ciliopathy that involves mutations in BBS proteins, which are known to be heavily involved in IFT. This condition can cause rod-cone dystrophy, polydactyly, hypogonadism and renal anomalies (23). Other ciliopathies include Meckel-Gruber syndrome and Joubert syndrome (24), both of these ciliopathies can cause craniofacial defects, the majority of common features of these facial abnormalities include defects in the lip and variations in the width of the mid-facial area (25).

\section{Primary cilia in the cell cycle}

The way in which the primary cilium must retract into a cell before the cell can enter mitosis is the most notable piece of evidence that primary cilia have an important role in preventing uncontrolled cell growth and the maintenance of an epithelial phenotype (26). The basal body acts as an anchor for the cilium to the cell body. This important ciliary component is formed from the mother centriole when it is released from the cilium and becomes a structure called the MTOC (2). It is hypothesized that ciliary resorption during the cell cycle could serve a key role in allowing the access of essential growth factors for cilia receptors, which are localized to the signaling hub that is the primary cilium. The resorption of the primary cilium during the cell cycle allows for the centrosome to detach from the basal body and to become the centrosome and the mitotic spindle, where they can then continue to carry out their role in the cell cycle (4). Further evidence is also emerging demonstrating the involvement of the primary cilia in cell cycle regulation. IFT88 serves a crucial role in primary cilia assembly and has also been demonstrated to serve a key role in the regulation of the $\mathrm{G}_{1}-\mathrm{S}$ transition in non-ciliated cells $(27,28)$. The centrosomal protein, nuclear distribution gene E homologue 1 (Nde1), has been indicated to have a negative regulatory role on cilia length (29). Nde1 is expressed in low levels during $\mathrm{G}_{0}$ and high levels during mitosis, and works by nucleating the primary cilium (30). Kim et al demonstrated that the knockdown of Nde1 in zebrafish caused an increased ciliary length with the suppression of cell division (31). With further evidence emerging demonstrating the association between cell cycle regulation and the primary cilium, there is an increased focus on the role of primary cilia in cancer.

\section{The loss of primary cilia expression in cancer}

Studies have previously shown a loss of cilia in a number of cancer types, including pancreatic and renal cancers $(32,33)$. Several ciliary-associated genes have been determined to be heavily dysregulated in cancer, including in clear renal cell carcinomas where there is a mutation in the von Hippel Lindau (VHL) tumor suppressor gene (33). The VHL gene is heavily involved in ciliogenesis, and the inheritance of this allele carries a high risk of developing clear cell renal carcinoma. Primary cilia have been indicated to be lost in patients with VHL disease (34). Notably the re-expression of VHL protein in clear cell renal carcinoma caused a restoration of cilia expression (35). Primary cilia have also been demonstrated to be frequently lost in human primary melanoma cells, compared with in primary melanocytes (36). Chefetz et al revealed that in ovarian cancer cells, the inhibition of aurora A kinase (AURA), a gene well known to be involved in cilia assembly, induced cell cycle arrest, providing another association between ciliogenesis and cell cycle regulation (37). Ciliogenesis has been indicated to be significantly suppressed in pancreatic cancer cells and pancreatic intraepithelial neoplasia (PanIN) lesions from human pancreatic ductal adenocarcinoma (PDAC). Cilia were determined to be lost in the PanIN cells of three different mouse models of PDAC (32). 


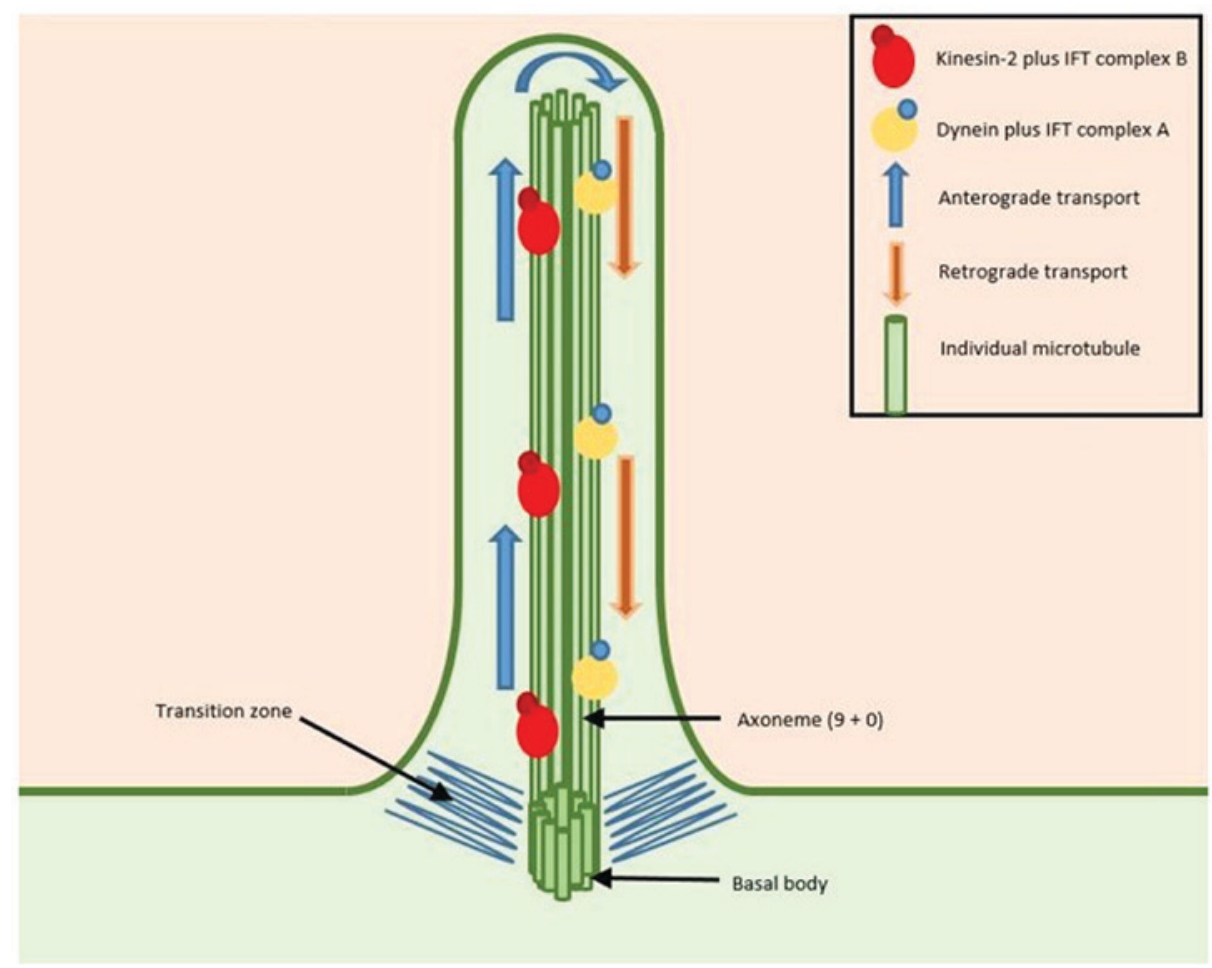

Figure 1. Structure of the primary cilium. The primary cilium is a microtubule-based organelle that is formed around an axoneme structure composed of 9 outer microtubule doublets. The cilium is formed in a process termed IFT. Anterograde transport channels ciliary proteins from the bottom of the cilium to its tip; the reverse can also occur in retrograde transport, which allows disassembly of the cilium. Kinesin-2, an anterograde motor protein transports ciliary proteins by forming a complex with IFT complex B, while the IFT complex A does the reverse by binding to dynein $1 \mathrm{~b}$ and channels ciliary proteins from the cilium to the cell body.

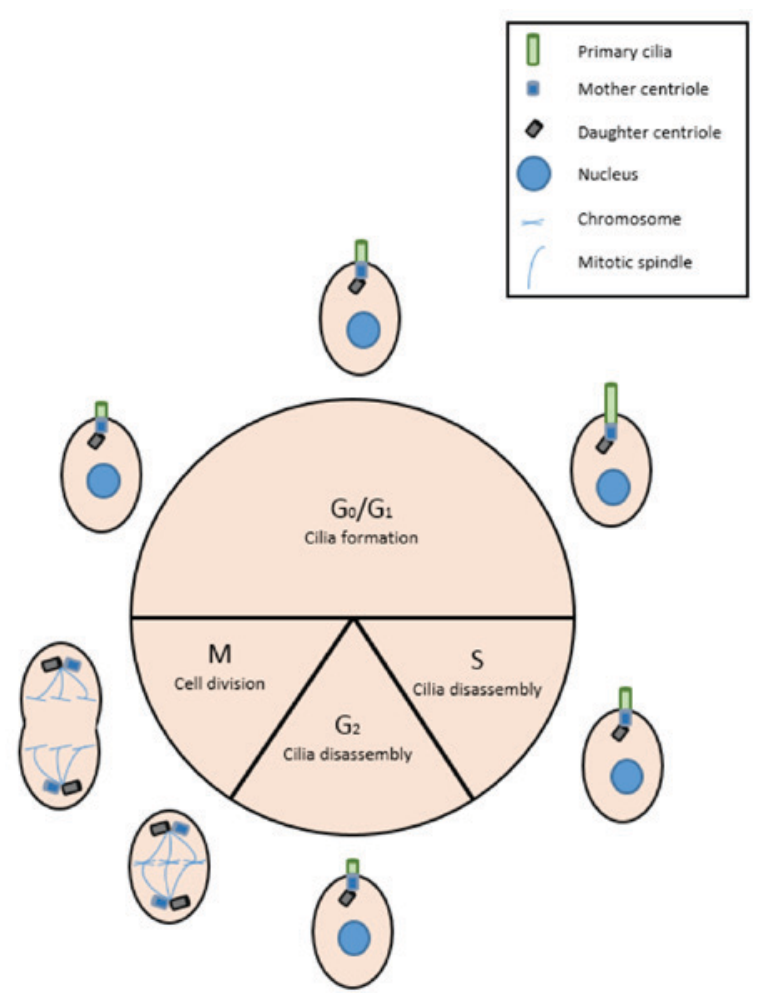

Figure 2. Primary cilia in the cell cycle. During $\mathrm{G}_{0}$ and $\mathrm{G}_{1}$ primary cilia are assembled and function as important signaling hubs. When the cell enters the $\mathrm{S}$ phase, ciliary disassembly begins with the resorption of the cilium. This allows the centrioles to detach from the basal body. The mother and daughter centrioles are duplicated and can move to the spindle poles where they become centrosomes and the mitotic spindle, and can now carry out their role in the cell cycle.
Egeberg et al indicated that a loss of primary cilia in ovarian cancer cells correlated with disrupted hedgehog and platelet derived growth factor receptor $\alpha$ (PDGFR $\alpha)$ signaling (38). The study indicated that the overexpression and the centrosomal localization of AURA on the ovarian surface epithelium dysregulates hedgehog signaling, disrupts the normal epithelial function of these ovarian cells leading to the eventual onset of tumor formation. The overexpression and the localization of AURA to the centrosome in ovarian surface epithelial cells indicated that AURA was involved in increasing the rate of ciliary disassembly, whilst also inhibiting the process of cilia formation (38). Emoto et al investigated the potential correlation between the presence/absence of primary cilia and the prognosis of PDAC. Primary cilia were determined to be present in $25 \%$ of cancer cells from patients with PDAC (39). The study demonstrated that the patients who were primary cilia-positive had a higher rate of lymph node metastasis and, thus, a poorer prognosis. Although these data are contrary to emerging evidence wherein primary cilia were demonstrably absent in numerous cancer cell lines, they raise the possibility of primary cilia being used as a hallmark of aggressive PDAC (39). As cellular proliferation is known to affect the presence of cilia, it is important to note that renal and pancreatic cancer cells do not contain primary cilia, independent of any decreases or increases in Ki67 staining (a marker of cell proliferation). This would support the idea that the loss of cilia in these cancer types is not associated with the rate of cell proliferation $(32,40)$, supporting the association between the primary cilium and carcinogenesis. 


\section{Primary cilia and ERK/MAPK signaling in cancer}

Mitogen-activated protein kinases (MAPKs), also known as extracellular signal-regulated kinases (ERKs), are a family of signaling proteins that are involved in cell differentiation, proliferation and development. Due to their important role in maintaining cellular homeostasis, their disregulation has been indicated in a number of cancer types (41). Within the ERK/MAPK signaling family, the components can be further categorized into four families: The MAPK classical pathway, the c-Jun N-terminal kinase (JNK), the big mitogen-activated protein kinase-1, and the p38 family (42). Briefly, the pathway involves Ras activating Raf kinase, which causes the phosphorylation of MAPK kinase (MEK)1/2, and leads to the phosphorylation and activation of ERK1/2 (41). It is the upregulation of ERK1/2 that contributes to cancer development, and usually occurs due to the overexpression of receptor tyrosine kinases (42). ERKs also exert a tumor suppressing ability by promoting selective protein degradation (43). Another important component of the MAPK family is p38. Similar to JNKs, p38 is also activated by stress-associated stimuli. p38 is thought to contribute to cancer growth by inducing prostaglandin-endoperoxide synthase 2 , whilst also influencing tumor invasion by inducing the expression of matrix metalloproteinase, which regulates matrix remodelling (44). It is also capable of inducing hypoxia inducible factor, which is directly involved in angiogenesis and could contribute to tumor development (44). The JNK family is activated in response to stress and is involved in the regulation of apoptosis, cell proliferation and differentiation. JNK is heavily implicated in apoptotic signaling (45). It is thought that an acute activation of JNK is involved in cell proliferation, whilst a more continuous activation is associated with apoptosis (46). With these seemingly contradictory regulatory roles, establishing the exact contribution of JNKs in cancer has been difficult. Chen et al demonstrated that JNK is actively involved in tumor growth, whilst also being implicated in tumor inhibition, as JNK-1 knockout mice were indicated to inhibit tumor growth, whereas JNK-2 enhanced it (47).

A study has previously shown an involvement of ERK/MAPK disregulation with cilia due to the exposure to renal carcinogens (48). Radford et al demonstrated that renal carcinogens were capable of inducing a loss of primary cilia in renal epithelial cells independent of the cell cycle. As well as inducing ciliary loss, ERK/MAPK-associated signaling pathways were determined to be heavily dysregulated following the exposure to these carcinogens (48). Other evidence indicated that ERK signaling may serve a role in ciliary regulation demonstrated sub-cloned HK-2 cells with shorter cilia had increased ERK activation, whilst ERK inhibition caused protection against cilia loss following cisplatin treatment (a chemotherapeutic nephrotoxin/ciliary shortening agent) (49). Another study demonstrated that ERK inhibition blocked the elongation of cilia in Madin-Darby canine kidney cells, indicating an association between ERK signaling and the regulation of cilia (50). Schneider et al demonstrated that PDGFR $\alpha \alpha$ signaling is regulated through the primary cilium in NIH3T3 fibroblasts. PDGFR $\alpha$ activation was indicated to activate AKT and ERK1/2, whilst Tg737 mutant cells lacking normal cilia results in a failure of PDGFR $\alpha \alpha, \mathrm{MEK} 1 / 2$ and ERK1/2 activation (51).

\section{Primary cilia and hedgehog signaling in cancer}

Hedgehog signaling is heavily involved in the regulation of cell growth and differentiation. A significant association is considered to exist between the primary cilium and cancer via this pathway (Fig. 3) (52). Numerous cancer types, including basal cell carcinoma, breast, prostate and lung cancer, have been indicated to involve abnormal activation of the hedgehog pathway (53). Abnormal hedgehog signaling in cancer can occur via a number of possible mutations, including mutations causing a loss of Ptch1 function and Smo activation. A total of $90 \%$ of basal cell carcinomas have a mutation affecting Ptch1, and 10\% have an activation of Smo. (53). Studies in mice have indicated that cilia are capable of serving a role in both promoting and preventing cancer development via their mediation of the hedgehog pathway (13). This is possibly due to the positive and negative regulators of the hedgehog pathway being processed in the primary cilium (13). Hedgehog signaling in the primary cilium is regulated by Ptch1. In general, the hedgehog component Smo is activated following Shh binding to and inactivating Ptch1, as Ptch1 represses Smo. Evidence indicates that when Shh binds to Ptch1, Ptch1 moves out of the cilium (13). This allows the accumulation of Smo and the activation of target gene transcription through the Gli family (10). It is thought that when cilia are present, inhibitors of hedgehog components Smo and Gli will control hedgehog signaling, whilst if cilia are absent or dysfunctional, this control can be lost and can result in the overexpression of hedgehog signaling (13). Within the domains of the hedgehog signaling pathway, the primary cilium can have a dual role in preventing and promoting carcinogenesis. This dual mechanism was demonstrated via a cilia deletion in basal cell carcinoma inhibited tumor growth induced by an activated form of the upstream activator of Smo, whilst also indicating that the loss of cilia caused cancer progression to be induced by activated Gli2, a downstream transcription factor (13). This dual role by primary cilia in hedgehog signaling was confirmed in granule neuron precursors, which can give rise to malignant brain tumors. It was demonstrated that the removal of primary cilia inhibited the formation of the medulloblastoma when it was driven by Smo, whilst cilia removal was required for tumor growth when it was driven by Gli2 (54). From this evidence, it is clear that primary cilia and hedgehog signaling are associated with the carcinogenic process, either in a promoting or an inhibitory capacity depending on the initiating carcinogenic factor.

\section{Primary cilia and Wnt signaling in cancer}

Alterations in Wnt signaling have long been linked to numerous cancer types, including breast, prostate, lung and colorectal (55). The canonical aspect of the Wnt signaling pathway is mediated by $\beta$-catenin, which upon activation translocates to the nucleus (56). Adenomatous polyposis coli is a tumor suppressor, which is significantly associated with Wnt signaling, and it has been demonstrated to be implicated in $\beta$-catenin regulation (57). An increase in $\beta$-catenin expression 


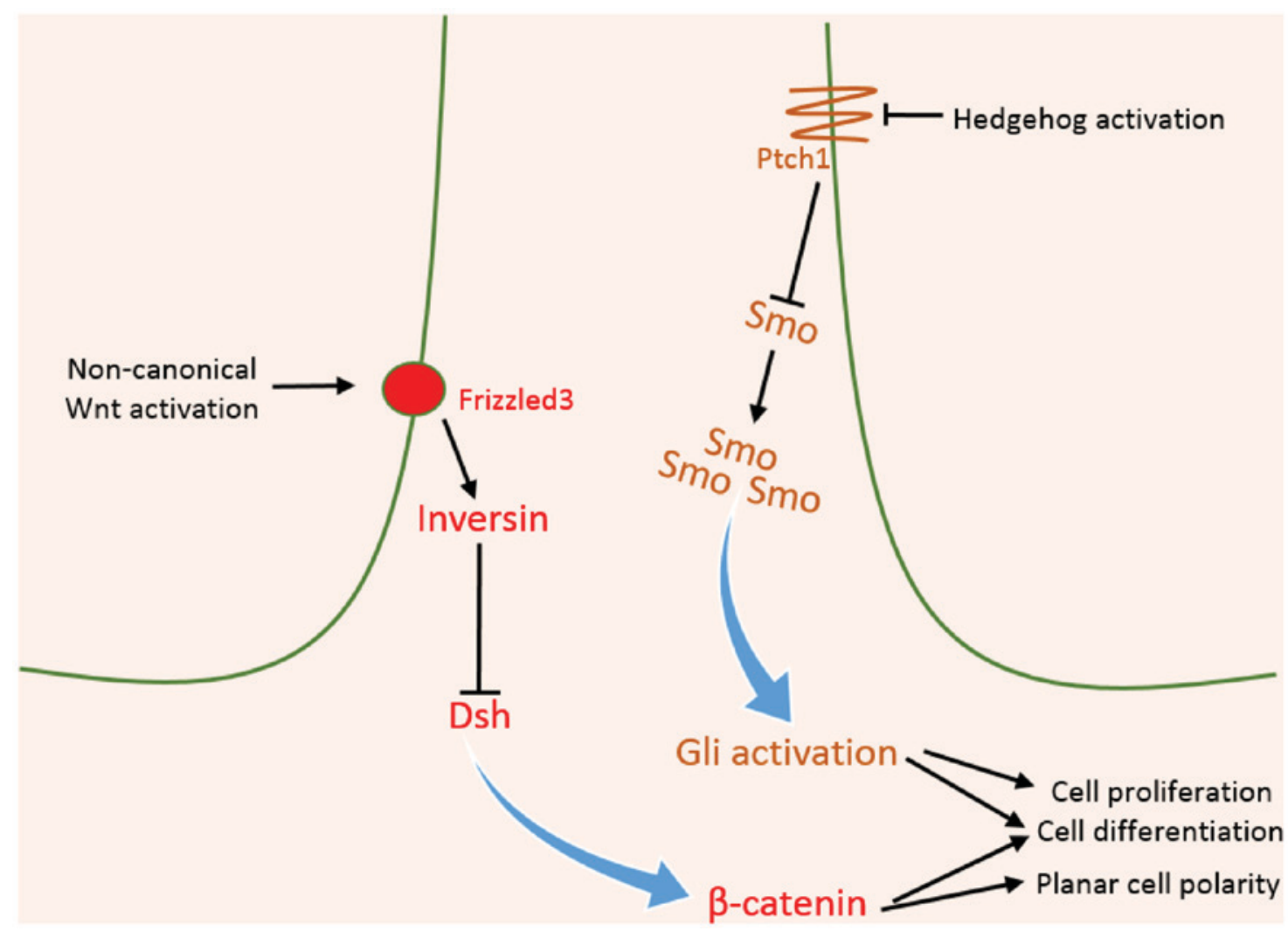

Figure 3. Hedgehog signaling and Wnt signaling in the primary cilium. The binding of hedgehog to Ptch1 results in Ptch1 inhibition and Smo accumulation as Ptch1 negatively regulates Smo. The accumulation of Smo leads to the activation of Gli family members and the inhibition of Gli repressors, eventually leading to cell proliferation. If the primary cilium is absent, Smo is incapable of activating Gli proteins leading to dysfunctional hedgehog signaling and increasing the possibility of cancer formation and development. Activation of the non-canonical Wnt signaling pathway by the stimulation of Frizzled 3 results in the inhibition of Dsh by Inversin, this inhibits $\beta$-catenin accumulation and ensures the maintenance of planar cell polarity. When the primary cilium is lost, it is possible that a switch from non-canonical to canonical Wnt signaling could occur, leading to $\beta$-catenin accumulation, the loss of PCP and uncontrolled cell proliferation.

levels correlates with a poor prognosis in a number of cancer types, including basal-like breast cancer (58). Lancaster et al demonstrated that impaired $\mathrm{Wnt} / \beta$-catenin signaling causes the induction of the cystic kidney disease NPHP, a type of ciliopathy (59). This type of medullary cystic disease is induced with a loss of function of the protein Jouberin. Jouberin helps to anchor the basal body to the plasma membrane, a mutation in this tectonic-like complex also occurs in Joubert syndrome, a disease with primary ciliary dysfunction (60). It is thought that Jbn, the gene that encodes for Jouberin, is required for a Wnt-associated response to renal injury repair (59).

Radford et al identified a number of dysregulated pathways following the exposure of renal epithelial cells (RPTEC/TERT1) to renal carcinogens ochratoxin A (OTA) and potassium bromate $\left(\mathrm{KBrO}_{3}\right)$ (48). These dysregulated pathways included the Wnt signaling and transforming growth factor- $\beta$-associated pathways. Notably, the study determined a notable association between the effect of these carcinogens and the primary cilium. OTA, a non-genotoxic carcinogen, and $\mathrm{KBrO}_{3}$, a genotoxic carcinogen, induced significant deciliation at $72 \mathrm{~h}$, whilst nifedipine, a non-carcinogenic renal toxin, had no impact on the expression of the primary cilium. The study identified the important effects that carcinogens have on renal primary cilia and raised the possibility that these deciliation events are serving a role in carcinogenesis (48).
The primary cilium serves a key role in controlling the non-canonical/PCP pathway (Fig. 3). Unlike cancer cells, fully differentiated epithelial cells are polarized (61). PCP allows epithelial cells to carry out their function by arranging them perpendicularly to the apico-basal axis (62). This PCP pathway is believed to serve a role in the maintenance of normal cellular homeostasis. A number of key components that help to regulate PCP have been indicated to be localized in the cilium (63). When FAT atypical cadherin 4, a protein involved in controlling PCP, is lost it can induce the formation of cysts in the kidney, indicating a role for proteins localized to the cilium in regulating PCP (63).

Inversin is an important component of the Wnt/PCP signaling pathway, it functions by targeting and degrading the cytoplasmic phosphoprotein Dsh, which inhibits $\beta$-catenin accumulation and, therefore, canonical Wnt signaling (64). Stimulation of the primary cilium via fluid flow has also been demonstrated to upregulate Inversin (65). Mutations in the gene encoding Inversin have been indicated to affect axis formation and induce cyst formation in PKD, both of which are processes significantly associated with cilia (66). By targeting and inhibiting the canonical, and not the non-canonical, pathway, it is possible that Inversin mediates a switch between these two pathways (64). If this is the case, the switch from the Wnt/PCP pathway to the $\mathrm{Wnt} / \beta$-catenin pathway would lead to uncontrolled cell proliferation and loss of PCP. Considering 
that the Wnt signaling pathway has an important role to serve in driving proliferation and controlling the maintenance of cellular morphology, its upregulation or dysfunction through the loss of primary cilia provides the strongest evidence associating primary cilia dysfunction and the induction of carcinogenesis.

Taking all this evidence together it can be considered that primary cilia, through their absence or dysfunction, contribute to the development of cancer via interference in well-established cancer signaling pathways, including Wnt, hedgehog and ERK/MAPK. Further studies are still required in order to fully understand the association between primary cilia and tumor formation.

\section{Acknowledgements}

Not applicable.

\section{Funding}

This review was supported by Science Foundation Ireland.

\section{Availability of data and materials}

Not applicable.

\section{Authors' contributions}

MH conceived and developed the outline and wrote the majority of the paper. IO contributed to the content of the paper. TM was involved in the conception, revising and final approval of the article. All authors also discussed and commented on the manuscript at all stages and contributed extensively to the work presented in this paper.

\section{Ethics approval and consent to participate}

Not applicable.

\section{Consent for publication}

Not applicable.

\section{Competing interests}

The authors declare that they have no competing interests.

\section{References}

1. Gluenz E, Höög JL, Smith AE, Dawe HR, Shaw MK and Gull K: Beyond 9+0: Noncanonical axoneme structures characterize sensory cilia from protists to humans. FASEB J 24: 3117-3121, 2010.

2. Lattao R, Kovács L and Glover DM: The centrioles, centrosomes, basal bodies, and cilia of drosophila melanogaster. Genetics 206 33-53, 2017.

3. Archer FL and Wheatley DN: Cilia in cell-cultured fibroblasts II. Incidence in mitotic and post-mitotic BHK 21-C13 fibroblasts. J Anat 109: 277-292, 1971.

4. Goto $\mathrm{H}$, Inoko A and Inagaki M: Cell cycle progression by the repression of primary cilia formation in proliferating cells. Cel Mol Life Sci 70: 3893-3905, 2013.

5. Pugacheva EN, Jablonski SA, Hartman TR, Henske EP and Golemis EA: HEF1-dependent Aurora A activation induces disassembly of the primary cilium. Cell 129: 1351-1363, 2007.
6. Cole DG, Diener DR, Himelblau AL, Beech PL, Fuster JC and Rosenbaum JL: Chlamydomonas kinesin-II-dependent intraflagellar transport (IFT): IFT particles contain proteins required for ciliary assembly in Caenorhabditis elegans sensory neurons. J Cell Biol 141: 993-1008, 1998.

7. Lechtreck KF: IFT-cargo interactions and protein transport in cilia. Trends Biochem Sci 40: 765-778, 2015.

8. Lancaster MA and Gleeson JG: The primary cilium as a cellular signaling center: Lessons from disease. Curr opin in Genet Dev 19: 220-229, 2009.

9. Grisanti L, Revenkova E, Gordon RE and Iomini C: Primary cilia maintain corneal epithelial homeostasis by regulation of the Notch signaling pathway. Development 143: 2160-2171, 2016.

10. Rohatgi R, Milenkovic L and Scott MP: Patched1 regulates hedgehog signaling at the primary cilium. Science 317: 372-376, 2007.

11. May-Simera HL and Kelley MW: Cilia, Wnt signaling, and the cytoskeleton. Cilia 1: 7, 2012

12. Corbit KC, Shyer AE, Dowdle WE, Gaulden J, Singla V, Chen MH, Chuang PT and Reiter JF: Kif3a constrains beta-catenin-dependent Wnt signalling through dual ciliary and non-ciliary mechanisms. Nat Cell Biol 10: 70-76, 2008.

13. Wong SY, Seol AD, So PL, Ermilov AN, Bichakjian CK, Epstein EH Jr, Dlugosz AA and Reiter JF: Primary cilia can both mediate and suppress Hedgehog pathway-dependent tumorigenesis. Nat Med 15: 1055-1061, 2009.

14. Corbit KC, Aanstad P, Singla V, Norman AR, Stainier DY and Reiter JF: Vertebrate smoothened functions at the primary cilium. Nature 437: 1018-1021, 2005.

15. Nozawa YI, Lin C and Chuang PT: Hedgehog signaling from the primary cilium to the nucleus: An emerging picture of ciliary localization, trafficking and transduction. Curr Opin Genet Dev 23: 429-437, 2013.

16. Ezratty EJ, Stokes N, Chai S, Shah AS, Williams SE and Fuchs E: A role for the primary cilium in Notch signaling and epidermal differentiation during skin development. Cell 145: 1129-1141, 2011.

17. Vladar EK Antic D and Axelrod JD: Planar cell polarity signaling: The developing cell's compass. Cold Spring Harb Perspect Bio 1: a002964, 2009.

18. Patel V, Li L, Cobo-Stark P, Shao X, Somlo S, Lin F and Igarashi P: Acute kidney injury and aberrant planar cell polarity induce cyst formation in mice lacking renal cilia. Hum Mol Genet 17: 1578-1590, 2008.

19. Tan AY, Blumenfeld J, Michaeel A, Donahue S, Bobb W, Parker T, Levine D and Rennert H: Autosomal dominant polycystic kidney disease caused by somatic and germline mosaicism. Clin Genet 87: 373-377, 2015.

20. Hajj P, Ferlicot S, Massoud W, Awad A, Hammoudi Y, Charpentier B, Durrbach A, Droupy S and Benoît G: Prevalence of renal cell carcinoma in patients with autosomal dominant polycystic kidney disease and chronic renal failure. Urology 74 : 631-634, 2009

21. Yoder BK, Hou X and Guay-Woodford LM: The polycystic kidney disease proteins, polycystin-1, polycystin-2, polaris, and cystin, are co-localized in renal cilia. J Am Soc Nephrol 13: 2508-2516, 2002.

22. Tory $\mathrm{K}$, Lacoste $\mathrm{T}$, Burglen $\mathrm{L}$, Morinière $\mathrm{V}$, Boddaert $\mathrm{N}$, Macher MA, Llanas B, Nivet H, Bensman A, Niaudet P, et al: High NPHP1 and NPHP6 mutation rate in patients with Joubert syndrome and nephronophthisis: Potential epistatic effect of NPHP6 and AHI1 mutations in patients with NPHP1 mutations. J Am Soc Nephrol 18: 1566-1575, 2007.

23. Forsythe $\mathrm{E}$ and Beales PL: Bardet-Biedl syndrome. Eur J Hum Genet 21: 8-13, 2013.

24. Waters AM and Beales PL: Ciliopathies: An expanding disease spectrum. Pediatr Nephrol 26: 1039-1056, 2010.

25. Cortes CR, Metzis V and Wicking C: Unmasking the ciliopathies: Craniofacial defects and the primary cilium. Wiley Interdiscip Rev Dev Biol 4: 637-653, 2015

26. Jones $\mathrm{C}$ and Chen P: Primary cilia in planar cell polarity regulation of the inner ear. Curr Top Dev Biol 85: 197-224, 2008.

27. Pazour GJ, Dickert BL, Vucica Y, Seeley ES, Rosenbaum JL, Witman GB and Cole DG: Chlamydomonas IFT88 and its mouse homologue, polycystic kidney disease gene tg737, are required for assembly of cilia and flagella. J Cell Biol 151: 709-718, 2000.

28. Robert A, Margall-Ducos G, Guidotti JE, Brégerie O, Celati C, Bréchot $\mathrm{C}$ and Desdouets C: The intraflagellar transport component IFT88/polaris is a centrosomal protein regulating G1-S transition in non-ciliated cells. J Cell Sci 120: 628-637, 2007. 
29. Inaba $H$, Goto $H$, Kasahara K, Kumamoto K, Yonemura S, Inoko A, Yamano S, Wanibuchi $\mathrm{H}, \mathrm{He} \mathrm{D}$, Goshima $\mathrm{N}$, et al: Ndel1 suppresses ciliogenesis in proliferating cells by regulating the trichoplein-Aurora A pathway. J Cell Biol 212: 409-423, 2016.

30. Maskey D, Marlin MC, Kim S, Kim S, Ong EC, Li G and Tsiokas L: Cell cycle-dependent ubiquitylation and destruction of NDE1 by CDK5-FBW7 regulates ciliary length. EMBO J 34 2424-2440, 2015.

31. Kim S, Zaghloul NA, Bubenshchikova E, Oh EC, Rankin S, Katsanis N, Obara T and Tsiokas L: Nde1-mediated inhibition of ciliogenesis affects cell cycle re-entry. Nat Cell Biol 13: 351-360, 2011.

32. Seeley ES, Carrière C, Goetze T, Longnecker DS and Korc M: Pancreatic cancer and precursor pancreatic intraepithelial neoplasia lesions are devoid of primary cilia. Cancer Res 69 : 422-430, 2009

33. Basten SG, Willekers S, Vermaat JS, Slaats GG, Voest EE, van Diest PJ and Giles RH: Reduced cilia frequencies in human renal cell carcinomas versus neighboring parenchymal tissue. Cilia 2: 2, 2013

34. Arjumand W and Sultana S: Role of VHL gene mutation in human renal cell carcinoma. Tumor Biol 33: 9-16, 2012

35. Esteban MA, Harten SK, Tran MG and Maxwell PH: Formation of primary cilia in the renal epithelium is regulated by the von Hippel-Lindau tumor suppressor protein. J Am Soc Nephrol 17: 1801-1806, 2006

36. Snedecor ER, Sung CC, Moncayo A, Rothstein BE, Mockler DC, Tonnesen MG, Jones EC, Fujita M, Clark RA, Shroyer KR and Chen J: Loss of primary cilia in melanoma cells is likely independent of proliferation and cell cycle progression. J Invest Dermatol 135: 1456-1458, 2015.

37. Chefetz I, Holmberg JC, Alvero AB, Visintin I and Mor G: Inhibition of Aurora-A kinase induces cell cycle arrest in epithelial ovarian cancer stem cells by affecting NFkB pathway. Cell Cycle 10: 2206-2214, 2011.

38. Egeberg DL, Lethan M, Manguso R, Schneider L, Awan A Jørgensen TS, Byskov AG, Pedersen LB and Christensen ST: Primary cilia and aberrant cell signaling in epithelial ovarian cancer. Cilia 1: 15, 2012.

39. Emoto K, Masugi Y, Yamazaki K, Effendi K, Tsujikawa H, Tanabe M, Kitagawa Y and Sakamoto M: Presence of primary cilia in cancer cells correlates with prognosis of pancreatic ducta adenocarcinoma. Hum Pathol 45: 817-825, 2013.

40. Yuan K, Frolova N, Xie Y, Wang D, Cook L, Kwon YJ, Steg AD, Serra R and Frost AR: Primary cilia are decreased in breast cancer: Analysis of a collection of human breast cancer cell lines and tissues. J Histochem Cytochem 58: 857-870, 2010.

41. Dhillon AS, Hagan S, Rath O and Kolch W: MAP Kinase signaling pathways in cancer. Oncogene 26: 3279-3290, 2007.

42. Burotto, M, Chiou VL, Lee JM and Kohn EC: The MAPK pathway across different malignancies: A new perspective. Cancer 120: 3446-3456, 2014.

43. Deschenes-Simard X, Gaumont-Leclerc MF, Bourdeau V, Lessard F, Moiseeva O, Forest V, Igelmann S, Mallette FA, Saba-El-Leil MK, Meloche S, et al: Tumor suppressor activity of the ERK/MAPK pathway by promoting selective protein degradation. Genes Dev 27: 900-915, 2013.

44. Wagner EF and Nebreda AR: Signal integration by JNK and p38 MAPK pathways in cancer development. Nat Rev Cancer 9: 537-549, 2009

45. Sanchez-Perez I, Murguia JR and Perona R: Cisplatin induces a persistent activation of JNK that is related to cell death. Oncogene 16: 533-540, 1998.

46. Dhanasekaran DN and Reddy EP: JNK signaling in apoptosis. Oncogene 27: 6245-6251, 2008.

47. Chen N, She QB, Bode AM and Dong Z: Differential gene expression profiles of Jnk1- and Jnk2-deficient murine fibroblast cells. Cancer Res 62: 1300-1304, 2002.
48. Radford R, Slattery C, Jennings P, Blacque O, Pfaller W, Gmuender H, Van Delft J, Ryan MP and McMorrow T: Carcinogens induce loss of the primary cilium in human renal proximal tubular epithelial cells independently of effects on the cell cycle. Am J Physiol Renal Physiol 302: F905-F916, 2012.

49. Wang SX, Wei Q, Dong G and Dong Z: ERK-mediated suppression of cilia in cisplatin-induced tubular cell apoptosis and acute kidney injury. Biochim Biophys Act 1832: 1582-1590, 2013.

50. Kim JI, Kim J, Jang HS, Noh MR, Lipschutz JH and Park KM: Reduction of oxidative stress during recovery accelerates normalization of primary cilia length that is altered after ischemic injury in murine kidneys. Am J Physiol Renal Physiol 304: F1283-F1294, 2013

51. Schneider L, Clement CA, Teilmann SC, Pazour GJ, Hoffmann EK, Satir P and Christensen ST: PDGFRalphaalpha signaling is regulated through the primary cilium in fibroblasts. Curr Biol 15: 1861-1866, 2005.

52. Goetz SC, Ocbina PJ and Anderson KV: The primary cilium as a Hedgehog signal transduction machine. Methods cell biol 94: 199-222, 2009.

53. Hassounah NB, Bunch TA and McDermott KM: Molecular pathways: The role of primary cilia in cancer progression and therapeutics with a focus on Hedgehog signaling. Clin Cancer Res 18: 2429-2435, 2012.

54. Han YG, Kim HJ, Dlugosz AA, Ellison DW, Gilbertson RJ and Alvarez-Buylla A: Dual and opposing roles of primary cilia in medulloblastoma development. Nat Med 15: 1062-1065, 2009.

55. Wissmann C, Wild PJ, Kaiser S, Roepcke S, Stoehr R, Woenckhaus M, Kristiansen G, Hsieh JC, Hofstaedter F, Hartmann A, et al: WIF1, a component of the Wnt pathway, is down-regulated in prostate, breast, lung, and bladder cancer. J Pathol 201: 204-212, 2003.

56. MacDonald BT, Tamai K and He X: Wnt/beta-catenin signaling: Components, mechanisms and diseases. Dev cell 17: 9-26, 2009.

57. Polakis P: Wnt signaling in cancer. Cold Spring Harb Perspect Biol 4: a008052, 2012.

58. Khramtsov AI, Khramtsova GF, Tretiakova M, Huo D, Olopade OI and Goss KH: Wnt/beta-catenin pathway activation is enriched in basal-like breast cancers and predicts poor outcome. Am J Pathol 176: 2911-2920, 2010.

59. Lancaster MA, Louie CM, Silhavy JL, Sintasath L, Decambre M, Nigam SK, Willert K and Gleeson JG: Impaired Wnt-beta-catenin signaling disrupts adult renal homeostasis and leads to cystic kidney ciliopathy. Nat Med 15: 1046-1054, 2009.

60. Eley L, Gabrielides C, Adams M, Johnson CA, Hildebrandt F and Sayer JA: Jouberin localizes to collecting ducts and interacts with nephrocystin-1. Kidney Int 74: 1139-1149, 2008.

61. Rodriguez-Boulan E and Macara IG: Organization and execution of the epithelial polarity programme. Nat Rev Mol Cell Biol 15: 225-242, 2014.

62. Macara IG, Guyer R, Richardson G, Huo Y and Ahmed SM: Epithelial homeostasis. Curr Biol 24: 815-825, 2014.

63. Saburi S, Hester I, Fischer E, Pontoglio M, Eremina V, Gessler M, Quaggin SE, Harrison R, Mount R and McNeill H: Loss of Fat4 disrupts PCP signaling and oriented cell division and leads to cystic kidney disease. Nat Genet 40: 1010-1015, 2008

64. Simons M, Gloy J, Ganner A, Bullerkotte A, Bashkurov M, Krönig C, Schermer B, Benzing T, Cabello OA, Jenny A, et al: Inversin, the gene product mutated in nephronophthisis type II, functions as a molecular switch between Wnt signaling pathways. Nat Genet 37: 537-543, 2005.

65. Nguyen AM and Jacobs CR: Emerging role of primary cilia as mechanosensors in osteocyte. Bone 54: 196-204, 2013

66. Otto EA, Schermer B, Obara T, O'Toole JF, Hiller KS, Mueller AM, Ruf RG, Hoefele J, Beekmann F, Landau D, et al: Mutations in INVS encoding inversin cause nephronophthisis type 2 , linking renal cystic disease to the function of primary cilia and left-right axis determination. Nat Genet 34: 413-420, 2003.

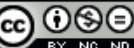

This work is licensed under a Creative Commons Attribution-NonCommercial-NoDerivatives 4.0 International (CC BY-NC-ND 4.0) License. 